\title{
ERGODICITY OF THE CARTESIAN PRODUCT
}

BY

\author{
ELIAS G. FLYT ZANIS
}

\begin{abstract}
. $h_{1}$ is an ergodic conservative transformation on a $\sigma$-finite measure space and $h_{2}$ is an ergodic measure preserving transformation on a finite measure space. We study the point spectrum properties of $h_{1} \times h_{2}$. In particular we show $h_{1} \times h_{2}$ is ergodic if and only if $h_{1} \times h_{2}$ have no eigenvalues in common other than the eigenvalue 1 . The conditions on $h_{1}, h_{2}$ stated above are in a sense the most general for the validity of this result.
\end{abstract}

1. Introduction. All measure spaces $(S, \Sigma, m)$ will be $\sigma$-finite in the sense of Halmos [7]. We consider transformations $b: S \rightarrow S$ that are measurable ( $A$ in $\Sigma$ implies $b^{-1}(A)$ in $\left.\Sigma\right)$, nonsingular $\left(m(A)=0\right.$ implies $\left.m\left(b^{-1}(A)\right)=0\right) . b$ is said to be conservative if $b^{-1}(A) \subseteq A$ implies $b^{-1}(A)=A$. We note that all statements about sets are made modulo sets of measure zero. Sometimes we replace the condition of being conservative on a $\sigma$-finite measure space by the stronger condition of being measure preserving $\left(m\left(b^{-1}(A)\right)=m(A)\right)$ on a probability space. A complex number $c$ is said to be an eigenvalue of $b$ if the equation $f(b(\cdot))=c f(\cdot)$ a.e. has a solution in $L_{\alpha}(S)$. $b$ is said to be ergodic if $b^{-1}(A)=A$ implies $m(A)=0$ or $m(S-A)=0$. In general the measurable sets that are invariant under $b$ (modulo sets of measure 0 ) form a sub-o-field and $b$ is ergodic if and only if that field is trivial.

Lemma 1. If $b$ is conservative then the eigenvalues of $b$ form a subgroup of the circle group. If $b$ is also ergodic then every eigenfunction is a constant a.e. in absolute value, and two eigenfunctions corresponding to the same eigenvalue differ only by a multiplicative constant. (It is not known whether the group of eigenvalues has to be countable.)

$H$ denotes a separable Hilbert space and $T$ a contraction in $H$, i.e. a bounded linear operator with $\|T\| \leq 1$. In $\$ 2$ we consider the eigenoperator equation

$$
T X(b(\cdot))=X(\cdot) \text { a.e., }
$$

where $X: S \rightarrow H$ is a measurable function. This type of equation was studied for the first time in [1]. We complete the solution by using some results from [8].

Received by the editors February 13, 1973.

AMS (MOS) subject classifications (1970). Primary 18A65.

Key words and phrases. Ergodic transformation, cartesian product, eigenoperation, Hilbert space, unitary operator. 
In $\$ 3$ we apply the results of $\$ 2$ to the following problem. $b_{1}$ is a conservative transformation on a $\sigma$ finite measure space $\left(s_{1}, \Sigma_{1}, m_{1}\right)$ and $b_{2}$ is a measure preserving transformation on a probability space $\left(s_{2}, \Sigma_{2}, m_{2}\right)$. We consider the eigenvalues of the cartesian product $b_{1} \times b_{2}$ defined on the measure space $\left(s_{1} \times S_{2}, \Sigma_{1} \times \Sigma_{2}, m_{1} \times m_{2}\right) . b_{1} \times b_{2}$ is also conservative and hence the eigenvalues have norm 1 . The eigenfunction equation $f\left(b_{1}\left(s_{1}\right), b_{2}\left(s_{2}\right)\right)=c f\left(s_{1}, s_{2}\right)$, where $f$ is in $L_{\infty}\left(S_{1}, S_{2}\right)$ can be put in the form (1).

In particular we consider the ergodicity of $b_{1} \times b_{2}$, and Theorem 2(ii) generalizes previous results ([3], [9], [12]). Theorem 2 allows us to construct (Example 1) conservative ergodic transformations that do not accept a finite invariant measure and have any given countable subgroup of the circle as their set of eigenvalues and whose restriction to a sub- $\sigma$-field is isomorphic to any given measure preserving transformation on a probability space. Also we show by an example taken from [10] that Theorem 2(iii) is not valid if we weaken the conditions on our transformations. However, a partial result still holds if $b_{2}$ is a measure preserving on an infinite ofinite measure space (Lemma 7). We note here that the results obtained are valid also for flows as well as more general group actions with the appropriate definitions.

The author would like to thank the referee for pointing out [10].

2. Eigenoperator equation. $b$ is a conservative transformation on a $\sigma$-finite measure space $(S, \Sigma, m) . H$ and $T$ are as above, and $X: S \rightarrow H$ is a measurable solution to the eigenoperator equation (1). The essential range $R(X) \subseteq H$ of $X$ is defined by " $X$ in $R(X)$ if for every neighborhood $N$ of $x$ we have $m\left(X^{-1}(N)\right)>0$ " We used the fact that if $b$ is conservative then given $A$ in $\Sigma, m(A)>0$, there exists a positive integer $n$ so that $m\left(b^{-n}(A) \cap A\right)>0$. Using this, we can prove the next lemma in the same way as the corresponding result in [1].

Lemma 2. (i) $R(X)$ is nonempty closed and invariant under $T$.

(ii) For a.a. $s$ in $S, X(s)$ in $R(X)$.

(iii) For each $x$ in $R(X)$ there exists a sequence of positive integers $\{n\}$ such that $T^{n} x \rightarrow x$.

Lemma 2(iii) characterizes $T$ completely in the subspace spanned by $P(x)=$ $\left\{T^{i} x, i=0,1,2, \ldots\right\}[8$, pp. 100-114]. We have

Lemma 3. (i) $T \mid \overline{\text { span }}(P(X))$ is a unitary operator baving discrete spectrum.

(ii) $T \mid \overline{\text { span }}(R(X))$ is a unitary operator baving discrete spectrum.

We note that (ii) follows from (i), using Lemma $2(\mathrm{i})$ and the property $\|T\| \leq 1$.

Let $v_{i}, i=1,2, \ldots$, denote an orthonormal basis of eigenvectors in $\overline{\operatorname{span}} R(X)$ given by the last lemma and $c_{i}, i=1,2, \ldots$, the corresponding eigenvalues. 
We set

$$
f_{i}(\cdot)=\left\langle v_{i} \cdot X(\cdot)\right\rangle, \quad i=1,2, \cdots
$$

Lemma 4. $f_{i}$ is an eigenfunction of $b$ corresponding to, the eigenvalue $c_{i}$ for each $i=1,2, \ldots$.

Proof.

$$
\bar{c}_{i} f_{i}(b(\cdot))=\bar{c}_{i}\left\langle v_{i}, X(b(\cdot))\right\rangle=\left\langle T^{*} v_{i}, X(b(\cdot))\right\rangle=\left\langle v_{i}, T X(b(\cdot))\right\rangle=\left\langle v_{i}, X(\cdot)\right\rangle \text { a.e. }
$$

Hence $f_{i}(b(\cdot))=c_{i} f_{i}(\cdot)$. We show that $f_{i}$ is not the trivial function. Since $v_{i}$ lies in $\overline{\operatorname{span}}(R(X))$ we have $\left\langle v_{i}, z\right\rangle \neq 0$ for some $z$ in $R(X)$. Hence $v_{i}$ defines a nonzero continuous function on $R(X)$. It follows from the definition of $R(X)$ that $f_{i}$ is not the trivial function. Q.E.D.

Theorem 1. (i) $b$ is a conservative transformation on a $\sigma$-finite measure space $(S, \Sigma, m) . H$ is a separable Hilbert space and $T$ is a contraction operator in $H$. Then the solutions to the eigenoperator equation,

$$
T X(b(\cdot))=X(\cdot) \text { a.e. in } S \text {, }
$$

where $X: S \rightarrow H$ is a measurable function are all the functions of the form

$$
X(\cdot)=\sum \bar{g}_{i}(\cdot) v_{i} \text { a.e. in } S,
$$

where $v_{i}$ is any orthonormal set of eigenvectors of $T$ and if $T v_{i}=c_{i} v_{i}$ then $g_{i}\left(h_{i}(\cdot)\right)=c_{i} g(\cdot)$. Also $\Sigma\left|g_{i}(\cdot)\right|^{2}<\infty$ a.e. in $S$ and the convergence is pointwise.

(ii) If $b$ is also ergodic then $\|X\|$ is constant a.e., and the convergence is in the essential sup norm.

The theorem above is a generalization of Lemma 1 under the assumption $\|T\| \leq 1$.

3. The cartesian product. $b_{1}$ is a conservative transformation on a $r$-finite measure space $\left(s_{1}, \Sigma_{1}, m_{1}\right)$ and $b_{2}$ is a measure preserving transformation on a probability space $\left(s_{2}, \Sigma_{2}, m_{2}\right)$. We consider the transformation $b_{1} \times b_{2}$ defined on the cartesian product $\left(s_{1} \times s_{2}, \Sigma_{1} \times \Sigma_{2}, m_{1} \times m_{2}\right)$ by $b_{1} \times b_{2}\left(s_{1}, s_{2}\right)=$. $\left(b_{1}\left(s_{1}\right), b_{2}\left(s_{2}\right)\right)$. We show first that $b_{1} \times b_{2}$ is conservative. We need the following

Lemma 5. $b$ is conservative if and only if $f(b(\cdot)) \leq f(\cdot)$ a.e. for a real valued measurable (or characteristic) function implies $f(b(\cdot))=f(\cdot)$ a.e.

Lemma 6. $b_{1} \times b_{2}$ is conservative. 
Proof. Let $f\left(s_{1}, s_{2}\right)$ be a characteristic function and $f\left(b_{1}\left(s_{1}\right), b_{2}\left(s_{2}\right)\right) \leq$ $f\left(s_{1}, s_{2}\right)$ a.e. By $[3$, p. 194] we have

$$
\int f\left(b_{1}\left(s_{1}\right), b_{2}\left(s_{2}\right)\right) d s_{2} \leq f\left(s_{1}, s_{2}\right) d s_{2} \text {. }
$$

where both sides are $m_{1}$ measurable. Setting $X(\cdot)=\int f\left(s_{1}, s_{2}\right) d s_{2}$ and using the measure preserving character of $b_{2}$, relation (i) becomes

$$
X\left(b\left(s_{1}\right)\right) \leq X\left(s_{1}\right) \text { a.e. in } s_{1} .
$$

By Lemma 5 we obtain $X\left(b\left(s_{1}\right)\right)=X\left(s_{1}\right)$ a.e. and (i) becomes also an equality. By $\left[3\right.$, p. 194] again, we have $f\left(b_{1}\left(s_{1}\right), b_{2}\left(s_{2}\right)\right)=f\left(s_{1}, s_{2}\right)$ a.e. in $s_{1} \times s_{2}$. The result follows from Lemma 5. Q.E.D.

Hence $b_{1} \times b_{2}$ has all its eigenvalues on the unit circle. Let $c$ be an eigenvalue of $b_{1} \times b_{2}$ and $f\left(b_{1}\left(s_{1}\right), b_{2}\left(s_{2}\right)\right)=c f\left(s_{1}, s_{2}\right)$ where $f$ is in $L_{\infty}\left(s_{1} \times s_{2}\right)$. The function $X: s_{1} \rightarrow L_{2}\left(s_{2}\right)$ is $m_{1}$-measurable by [2, p. 196, Lemma $\left.6 \mathrm{~b}\right]$ and the equation becomes

$$
\operatorname{TX}\left(b_{1}(\cdot)\right)=X(.) \text { a.e. in } S_{1} \text {, }
$$

where $T$ is the isometry in $L_{2}\left(S_{2}\right)$ defined by $T g(\cdot)=\bar{c} g\left(b_{2}(\cdot)\right)$ a.e. in $S_{2} \cdot$ By Theorem 1 we obtain

Theorem 2. $b_{1}, b_{2}$ are as above. $C=\left\{c_{i}\right\}, D=\left\{d_{j}\right\}$ are the corresponding groups of eigenvalues. We consider the groups $C \cap D$ and $C D$. Then

(i) $C D$ is the group of eigenvalues of $b_{1} \times b_{2}$.

(ii) The sub-o.field of $\Sigma_{1} \times \Sigma_{2}$ of the invariant sets of $b_{1} \times b_{2}$ is that generated by the functions of the form $\bar{u}\left(s_{1}\right) \cdot v\left(s_{2}\right)$, where $u, v$, are eigenfunctions of $b_{1}, b_{2}$ respectively corresponding to the same eigenvalue in $C \cap D$. $\{1\}$.

(iii) $b_{1} \times b_{2}$ is ergodic if and only if $b_{1}, b_{2}$ are both ergodic and $C \cap D=$

Example 1. In [2] or [4, Example 6.8] a general method is given for constructing ergodic transformations that do not accept a finite invariant measure and in [11] or [4, Example 6.8] a conservative and ergodic measure preserving transformation on a probability space is constructed having no eigenvalues except 1 . It is possible to combine these two techniques and obtain an ergadic transformation that does not accept a finite invariant measure and also that has no eigenvalues except 1. Denoting this transformation by $b_{1}$, we can take for $b_{2}$ any ergodic m.p.t. on a probability space and then $b_{1} \times b_{2}$ will be always ergodic, it will not accept a finite measure space and its restriction to a sub- $\sigma$-field will be isomorphic to the given $b_{2}$. Also it will have the same eigenvalues as $b_{2}$. 
Remarks. Concerning the validity of Theorem 2 (iii) under more general conditions on the transformations $b_{1}, b_{2}$ we note the following. Clearly the result is not valid if we do not assume $b_{1}$ to be conservative, as can be seen by taking $b_{1}$ to be the shift transformation on the integers. In fact for invertible transformations the only ergodic transformation that is not conservative is exactly this. Concerning the conditions on $b_{2}$ we note the following

Example 2. In [10]we are given an example of an ergodic transformation $b$ which is measure preserving on an infinite o-finite measure space and for which

(i) $b \times b$ is ergodic,

(ii) $b \times b \times b$ is not ergodic.

It follows from (i) that $b$ has no eigenvalues. Setting $b_{1}=b \times b$ and $b_{2}=b$ we note that they have no eigenvalues in common and yet $b_{1} \times b_{2}$ is not ergodic. In fact for the case where $b_{2}$ is ergodic and measure preserving on an infinite ofinite measure space we note that the induced transformation in $L_{2}\left(S_{2}\right)$ has no eigenvalues and using the same technique as above we obtain

Lemma 7. $h_{1}$ is a conservative ergodic transformation on a $\sigma$-finite measure space and $b_{2}$ is an ergodic measure preserving transformation on an infinite $\sigma-f i$ nite measure space. Then $b_{1} \times b_{2}$ is conservative; and if $A$ is an invariant subset of $b_{1} \times b_{2}$ then the function on $s_{1}$ defined by $m_{2}\left(s_{2}:\left(s_{1}, s_{2}\right)\right.$ in $\left.A\right)$ bas infinite value over a set of nonzero measure in $S_{1}$. In particular, there are no invariant sets of finite measure.

The result above must be rather limited as we use the isometry induced by $b_{2}$ in $L_{2}\left(S_{2}\right)$ and even the ergodicity of $b_{2}$ is not an invariant of this isometry. We could obtain complete information for the general cartesian product problem if we could solve the eigenoperator equation where $T$ is assumed only bounded though not necessarily an isometry. It can be shown that in this case the essential range of $X(\cdot)$ consists of nonwandering points of $T$. If the Hilbert space is finite dimensional this makes $T$ totally bounded and reduces to the case considered. The solution is not known if the Hilbert space is infinite dimensional.

\section{REFERENCES}

1. A. Beck, Eigenoperators of ergodic transformations, Trans. Amer. Math. Soc. 94 (1960), 118-129. MR 27\#2606.

2. R. V. Chac on, A class of linear transformations, Proc. Amer. Math. Soc. 15 (1964), 560-564. MR 29\#2362.

3. N. Dunford and J. T. Schwartz, Linear operators. I: General theory, Pure and Appl. Math., vol. 7, Interscience, New York, 1958. MR 22 \#8302.

4. N. A. Friedman, Introduction to ergodic theory, Van Nostrand, Princeton, N. J., 1970.

5. H. Fürstenberg, Disjointness in ergodic theory, minimal sets and a problem in Diophantine approximations, Math. Systems Theory 1 (1967), 1-49. MR 35 \#4369. 
6. P. R. Halmos, Lectures on ergodic theory, Publ. Math. Soc. Japan, no. 3, Math. Soc. Japan, Tokyo, 1956; reprint, Chelsea, New York, 1960. MR 20 \#3958.

7. - Measure theory, Van' Nostrand, Princeton, N. J., 1950. MR 11, 504.

8. K. Jacobs, Lecture notes on ergodic theory. Vol. 1, Mathematisk Institut, Aarhus Universitet, Aarhus, 1962/63, pp. 1-207. MR 28 \#3138.

9. B. Kronfeld, Ergodic transformations and independence, Glasnik Math. Ser. III S(25) (1970), 221-226. MR 43 \#2189.

10. S. Kakutani and W. Parry, Infinite measure preserving transformations with mixing, Bull. Amer. Math. Soc. 69 (1963), 752-756. MR 27 \#3776.

11. D. S. Ornste in, On invariant measures, Bull. Amer. Math. Soc. 66 (1960), 297-300. MR $26 \# 3872$.

12. W. Parry and F. Hahn, Some characteristic properties of dynamical systems with quasi-discrete spectra, Math. Systems Theory 2 (1968), 179-190. MR 37 \#6435.

DEPARTMENT OF MATHEMATICS, PIERCE COLLEGE, ATHENS, GREECE 